\title{
Measuring the impact of cancer: a comparison of non-Hodgkin lymphoma and breast cancer survivors
}

\author{
Catherine M. Crespi • Sophia K. Smith • \\ Laura Petersen • Sheryl Zimmerman • Patricia A. Ganz
}

Received: 10 July 2009/Accepted: 6 November 2009/Published online: 6 December 2009

(C) The Author(s) 2009. This article is published with open access at Springerlink.com

\begin{abstract}
Introduction Self-report instruments such as the Impact of Cancer (IOC) are designed to measure quality of life (QOL) impacts that cancer survivors attribute to their cancer experience. Generalizability of QOL findings across distinct diagnostic categories of survivors is untested. We compare measurement of the impact of cancer using the IOC instrument in breast cancer (BC) survivors $(n=1,188)$ and non-Hodgkin lymphoma (NHL) survivors $(n=652)$. Methods A registry-based sample of NHL survivors completed the IOC questionnaire and the FACT-G, FACT-LYM, Medical Outcomes Study (MOS) SF-36, Post-Traumatic Stress Disorder Checklist-Civilian Version, Post-Traumatic
\end{abstract}

Electronic supplementary material The online version of this article (doi:10.1007/s11764-009-0106-1) contains supplementary material, which is available to authorized users.

\section{M. Crespi $(\triangle)$}

Department of Biostatistics, School of Public Health,

University of California Los Angeles,

CHS 51-254, Box 951772, Los Angeles, CA 90095-1772, USA

e-mail: ccrespi@ucla.edu

L. Petersen · P. A. Ganz

Division of Cancer Prevention and Control Research,

Jonsson Comprehensive Cancer Center,

University of California Los Angeles,

Los Angeles, CA, USA

S. K. Smith $\cdot$ S. Zimmerman

Sheps Center for Health Services Research,

University of North Carolina at Chapel Hill,

Chapel Hill, NC, USA

P. A. Ganz

School of Medicine, University of California Los Angeles,

Los Angeles, CA, USA
Growth Inventory and MOS Social Support scales. IOC responses of the NHL survivors were subjected to de novo scaling to identify impact domains for comparison to IOC version 2 (IOCv2) domains, which were previously developed based on BC survivor responses. Concurrent validity was assessed by correlating the IOCv2 scales with the other measures. IOCv2 scores were compared between the $\mathrm{BC}$ and NHL survivor samples.

Results The $\mathrm{BC}$ and NHL survivors exhibited similar impact domains and had factor structures that were largely congruent. The concurrent validity analysis revealed patterns of association that supported the interpretation and validity of the IOCv2 scales. Differences in IOCv2 scores between the BC and NHL groups suggested differential impacts in distinct survivor groups that could be detected using the IOCv2.

Conclusion The results suggest that the IOCv2 measures common and important survivor concerns and support its generalizability to the broader long-term cancer survivor population.

Implications for Cancer Survivors Instruments such as the IOCv2 can provide valid assessment of QOL impacts in long-term cancer survivors, facilitating the characterization of these impacts and development of appropriate interventions.

Keywords Breast cancer-Cancer survivor.

Non-Hodgkin lymphoma $\cdot$ Quality of life

\section{Introduction}

There is a large and growing population of cancer survivors [1], with an expanding literature documenting impacts of cancer among survivors across physical, psychological, 
emotional, social and spiritual domains [2-6]. The need for research on health-related quality of life (HRQOL) in this growing at-risk population was recently highlighted by the Institute of Medicine [7]. Valid assessment of HRQOL in cancer survivors is a prerequisite for characterizing and monitoring these impacts and developing appropriate interventions. However, standardized measures of HRQOL often fail to address the unique concerns reported by longterm survivors. For example, many survivors experience health and body image concerns that are specifically cancer-related [8-10]. In addition, research with cancer patients has increasingly recognized that a large proportion report positive life changes or personal growth as a result of their illness, a construct referred to as benefit finding [11-16]. Generic HRQOL measures are generally not designed to capture these domains. This has created a need to develop targeted instruments measuring domains important to the well-being of cancer survivors [17-20].

Several instruments have been developed specifically to measure the concerns of long-term cancer survivors. An early example is the Quality of Life-Cancer Survivors (QOL-CS) scale [21], an instrument adapted from existing broad-based HRQOL instruments composed predominantly of generic items. The Quality of Life in Adult Cancer Survivors (QLACS) scale $[22,23]$ is a more recent instrument covering five cancer-specific domains (Financial Problems, Benefits, Distress-Family, Appearance, DistressRecurrence) and seven generic domains (Negative Feelings, Positive Feelings, Cognitive Problems, Pain, Sexual Interest, Energy/Fatigue, Sexual Function, Social Avoidance). Targeted, construct-specific instruments for cancer survivors have also been developed. For example, the Breast Cancer Prevention Trial (BCPT) Symptom Checklist assesses physical effects of medical intervention to prevent and treat breast cancer [24, 25], and several scales measure fear of cancer recurrence $[26,27]$. Construct-specific scales such as these are generally designed to serve as adjuncts to, or modules in, other HRQOL instruments.

With the goal of developing a comprehensive instrument specifically designed to measure HRQOL impacts that long-term survivors ascribe to their cancer experience and with funding from the National Cancer Institute's Office of Cancer Survivorship, members of our group and others have developed the Impact of Cancer (IOC) scale [28-30]. The IOC measures both negative impacts and the positive life changes reported by many survivors, such as a finding of meaning or purpose, which generic HRQOL instruments are generally not designed to capture [31-33]. The IOC was developed through a standard psychometric approach (qualitative interviews with long-term survivors for item generation followed by item reduction, cognitive testing and additional reduction to a pool of 81 items), leading to an initial scale identification using data from 193 survivors of breast, prostate, colorectal and lymphatic cancers [28, 29]. Recently, more comprehensive scaling using data from 1,188 breast cancer (BC) survivors was conducted [30]. This effort included de novo scaling of the 81-item pool, split-sample cross-validation and evaluation of internal consistency and construct and concurrent validity, yielding the Impact of Cancer version 2 (IOCv2) scales (instrument available from the first author) [30], with 37 items composing positive and negative summary scales (including eight subscales) and 10 items measuring employment and relationship concerns.

To be widely useful, instruments measuring HRQOL in cancer survivors must be valid across different cancer diagnoses. The IOCv2 scales demonstrated excellent internal consistency and validity, but proof of their generalizability is limited due to testing only in female BC survivors who were 5-10 years post-diagnosis. Would these same scales be confirmed among survivors with a different cancer diagnosis, gender distribution and treatment trajectory? In particular, would another survivor group identify similar impact domains and have scale scores that are equally valid measurements of the underlying constructs? Investigation of these issues is necessary to establish reliable and well-validated scales to measure the psychosocial impacts of cancer across the spectrum of diagnoses and survivorship stages. This study was undertaken to examine the reliability and validity of the IOCv2 scales in an independent sample of 652 disease-free non-Hodgkin lymphoma (NHL) survivors, and to compare results for the $\mathrm{BC}$ and NHL survivors to evaluate the generalizability of the IOCv2 across survivor samples.

\section{Participants and methods}

\section{Participants}

NHL survivors were identified through the Duke University and University of North Carolina at Chapel Hill Lineberger tumor registries in November 2004 as previously described [34]. Patients were eligible if diagnosed with NHL, $\geq 19$ years old at diagnosis, and $\geq 2$ years post-diagnosis. Prospective participants were mailed a self-administered survey that included the 81 IOC items and other measures described below. Of the 1195 eligible survivors who were assumed to have received a mailed survey, $886(74 \%)$ returned survey materials. Participating survivors were less frequently African American and older at diagnosis and study enrollment [34]. Patients reporting active disease or with unknown recurrence status $(n=234)$ were excluded to limit the analysis to diseasefree survivors.

The BC survivors were members of the Life After Cancer Epidemiology cohort [35], recruited primarily from 
the Kaiser Permanente Northern California and Utah cancer registries. Patients were eligible if they were diagnosed at age 18-79 with a primary breast cancer (Stage $1 \geq 1 \mathrm{~cm}, 2$, 2A) within 39 months of enrollment, had no history of other cancer within five years of enrollment, had completed treatment (except for adjuvant hormonal therapy), and were recurrence-free at enrollment. Of 5656 women presumed to meet the LACE eligibility criteria, 2614 (46\%) agreed to participate. Responders were closer to their diagnosis date and older than nonresponders but did not differ in severity of cancer or treatment [35]. Subsequent review resulted in 293 exclusions. Participants completed the 81 IOC items as part of a mailed self-administered resurvey April 2006January 2007. The IOC questionnaire was returned by 1286 of 1805 women (71\%). Respondents were older at diagnosis and enrollment, more likely to be white, and had higher levels of education than nonrespondents [30]. Women with recurrent or new primary disease or unknown status in this regard were excluded from our study sample $(n=98)$.

Institutional review board approval was obtained at all institutions participating in the study and written informed consent was obtained from each participant.

\section{Measures}

The full IOC questionnaire [28] comprises 81 items that present statements regarding specific impacts of cancer to which respondents indicate their level of agreement from 1 (strongly disagree) to 5 (strongly agree). Seventy items apply to all survivors, 3 to currently employed respondents, 4 to respondents not currently partnered, and 4 to currently partnered respondents. The IOCv2, a scaled instrument using 47 of these items [30] is provided in the appendix online.

The Functional Assessment of Cancer Therapy: General (FACT-G) is a general HRQOL measure for patients receiving cancer treatment that yields a total score and subscales for physical, functional, social/family and emotional well-being [36]. FACT-LYM is a diagnosis-specific measure for NHL patients [37].

The Medical Outcomes Study (MOS) SF-36 is a generic patient-assessed health outcome measure yielding eight subscales and physical and mental health summary scores [38].

The Post-Traumatic Stress Disorder Checklist-Civilian Version (PCL-C) presents a self-report symptom checklist that mirrors criteria for a formal diagnosis of post-traumatic stress disorder [39, 40]. Instructions were modified to key symptoms to lymphoma diagnosis and treatment as the stressor of interest. Survivors rated 17 symptoms on intensity from 1 (not at all) to 5 (extremely bothersome).

The Posttraumatic Growth Inventory (PTGI) assesses positive outcomes reported by persons who have experienced traumatic events and yields a total scale and five subscales [41, 42].

The MOS Social Support Score assesses perceived availability of social support, with a total scale and four subscales pertaining to particular functions served by interpersonal relationships [43].

\section{Statistical analysis}

The goals of the analyses were to perform an independent psychometric evaluation of the IOC in the NHL sample and to compare these results with those from the BC survivors. First we conducted a de novo exploratory factor analysis on the NHL survivor data using the 70 IOC items applicable to all survivors, closely mirroring the scaling process previously used for the BC survivor sample [30]. Employment and relationship items, which were scaled separately for the BC sample, were also not included in this factor analysis for the NHL sample. Factors were extracted using principal components, the number of factors was selected using eigenvalue $>1$ and scree plots, and promax rotation was performed. After an initial factor analysis, items with factor loadings $<0.50$ or not associated with a clearly identifiable factor were dropped and the factor analysis was repeated to produce a set of factors for comparison to the IOCv2 scales obtained from the BC survivor analysis.

Next, we assessed whether the 37-item, 8-factor structure of the IOCv2 positive and negative subscales exhibited by the BC survivors was reproduced in the NHL survivor sample using the targeted rotation method [44], which compares the factor structure of a second sample (NHL survivors) to a reference structure (BC survivors) by extracting the hypothesized number of factors from the second sample and calculating congruence coefficients, which compare two sets of factor loadings in terms of pattern and magnitude [44, 45].

Concurrent validity was evaluated by forming a priori hypotheses about patterns of association and correlating the IOCv2 scores with HRQOL (FACT, SF-36), PCL-C, PTGI and Social Support scores. We hypothesized that the IOCv2 positive scales would be uncorrelated with the HRQOL measures (which are not designed to capture positive impacts) and positively correlated with the PTGI total and subscales (which similarly measure personal growth associated with a traumatic event). We expected an overall pattern of moderate to strong correlation between the IOCv2 negative scales and the HRQOL scales. We hypothesized that the IOCv2 negative summary score would be strongly correlated with aggregate measures of negative impacts (FACT-G, FACT-LYM, SF-36 Physical and Mental, PCL-C Total). We further hypothesized that IOCv2 Body Change Concerns would be correlated with scales measuring physical limitations, that IOCv2 Life 
Interferences would be correlated with scales measuring functional limitations, and that IOCv2 Worry would be correlated with FACT-G Emotional. We expected IOCv2 Employment Concerns to be moderately correlated with HRQOL measures, and IOCv2 Relationship Concerns to be negatively correlated with the FACT-G Social/Family and Social Support scales.

When evaluating the quantitative significance of correlations, we considered $|r|<0.30$ to indicate weak association, $0.30 \leq|r|<0.45$ moderate, $0.45 \leq|r|<0.60$ substantial and $|r| \geq$ 0.60 high [46]. We used $P<0.005$ for statistical significance to account for the large sample size and multiple comparisons. All $P$-values and significance tests were two-sided.

Internal consistency reliability was measured using Cronbach's alpha [47]. We obtained mean scale scores adjusted for age and years since diagnosis and tested for differences in adjusted means using the SAS GLM procedure. All analyses were conducted using SAS 9.1.

\section{Results}

\section{Sample characteristics}

The NHL group included similar numbers of men and women, in contrast to the exclusively female BC survivors (Table 1). Comparisons revealed significant differences between the groups on all characteristics except education and income. On average, the $\mathrm{BC}$ survivors were diagnosed at an older age and were older at time of survey. Mean interval since diagnosis was longer in the NHL survivors, who also had a broader range of interval. The NHL survivors reported about one more comorbidity on average than the $\mathrm{BC}$ group. Despite statistically significant differences in race/ethnicity and marital status, both groups were predominantly white and partnered.

\section{De novo factor analysis}

Table 2 presents results of the de novo exploratory factor analysis of the IOC items for the NHL sample. Eight domains emerged that were largely congruent with the eight domains found in the IOCv2 scaling on the BC survivor data. In particular, factors for Altruism/Empathy, Health Awareness, Meaning of Cancer, Positive Self-Evaluation, Appearance Concerns and Worry emerged that were either identical to the IOCv2 in item content or differed by one item. Whereas Body Change Concerns and Life Interferences emerged as distinct factors in the $\mathrm{BC}$ survivor data, these two constructs emerged as a single factor in the NHL sample. However, all Body Change Concern items in the IOCv2 were also identified in the NHL sample, and five of the seven life interference items in the IOCv2 also appeared in the NHL sample.
The NHL analysis also yielded a three-item scale that did not emerge in the $\mathrm{BC}$ sample but which was identical to the Positive Outlook subscale identified in the first scaling of the IOC in a sample of 193 survivors of breast, colorectal, lymphatic and prostate cancers [28].

\section{Reproducibility of factor structure}

The 8 -factor structure of the IOCv2 positive and negative subscales exhibited by the BC survivor sample was wellreproduced in the NHL survivor sample, as evidenced by high congruence coefficients (Table 3 ). All item congruences exceeded 0.90 with the exception of two items on the Life Interferences subscale. Despite these lower values, the factor congruence for Life Interferences was 0.88. Other factor congruences ranged from $0.95-0.98$.

\section{Concurrent validity}

Table 4 summarizes the NHL survivor scores on the FACT, SF-36, PCL-C, PTGI and Social Support scales, and presents the correlations of these scores with the IOCv2 positive scales. Table 5 presents correlations with the IOCv2 negative and employment and relationship concern scales.

The observed patterns of association were largely consistent with prior hypotheses. There was a striking lack of correlation between the IOCv2 positive scales and the FACT-G, FACT-LYM and SF-36, supporting the distinctive content of the IOCv2 positive domains from these scales. The PCL-C and Social Support scales were also uncorrelated with the IOCv2 positive scales, indicating that the IOCv2 positive constructs were unrelated to either posttraumatic stress symptoms or perceived availability of particular relationships. Strong or substantial correlations were observed between the PTGI and IOCv2 Altruism/ Empathy, Meaning of Cancer and Positive Self-Evaluation, as well as the IOCv2 Positive Impact Summary, as expected due to their similar goal of measuring personal growth ascribed to a traumatic experience. There was lesser correlation with IOCv2 Health Awareness, consistent with the lack of health focus of the PTGI.

As expected, there was an overall pattern of moderate to strong correlation between the IOCv2 negative scales and the FACT-G, FACT-LYM and SF-36, with higher correlations evident for the FACT-G and FACT-LYM, which are cancer-specific, than for the SF-36, which is not. The strongest correlation was between the IOCv2 Negative Impact Summary score and the FACT-LYM, which is specifically designed to measure negative HRQOL symptoms in lymphoma survivors. The IOCv2 Negative Impact Summary score was also correlated with the other aggregate measures of negative impacts (FACT-G, SF-36 Physical and Mental, PCL-C). Particularly striking were strong correlations 
Table 1 Characteristics of the non-Hodgkin lymphoma and breast cancer survivor samples

\begin{tabular}{|c|c|c|c|c|c|}
\hline \multirow[b]{2}{*}{ Characteristic } & \multicolumn{2}{|c|}{ NHL survivors } & \multicolumn{2}{|c|}{ BC survivors } & \multirow[t]{2}{*}{$p$} \\
\hline & No. & $\%$ & No. & $\%$ & \\
\hline Total No. of respondents & 652 & & 1188 & & \\
\hline Sex & & & & & $<0.001$ \\
\hline Male & 329 & 50 & & & \\
\hline Female & 323 & 50 & 1188 & 100 & \\
\hline Race & & & & & $<0.001$ \\
\hline White & 565 & 87 & 958 & 83 & \\
\hline African American & 51 & 8 & 36 & 3 & \\
\hline Hispanic & 9 & 1 & 58 & 5 & \\
\hline Asian/Pacific Islander & 2 & 0.3 & 60 & 5 & \\
\hline Other/more than one & 25 & 4 & 46 & 4 & \\
\hline Marital status & & & & & $<0.001$ \\
\hline Married/living with partner & 518 & 80 & 799 & 68 & \\
\hline Widowed/divorced/separated/single & 133 & 20 & 384 & 32 & \\
\hline Income & & & & & 0.769 \\
\hline$<\$ 60,000$ & 331 & 56 & 612 & 56 & \\
\hline$\geq \$ 60,000$ & 255 & 44 & 486 & 44 & \\
\hline Education & & & & & 0.163 \\
\hline High school or less & 179 & 28 & 290 & 24 & \\
\hline Some college & 205 & 32 & 423 & 36 & \\
\hline College graduate or higher & 259 & 40 & 471 & 40 & \\
\hline Employment & & & & & 0.015 \\
\hline Employed & 263 & 41 & 412 & 35 & \\
\hline Retired/unemployed & 381 & 59 & 763 & 65 & \\
\hline Age, years & & & & & $<0.001$ \\
\hline $25-49$ & 115 & 18 & 63 & 5 & \\
\hline $50-64$ & 235 & 36 & 474 & 40 & \\
\hline $65-79$ & 237 & 36 & 536 & 45 & \\
\hline$\geq 80$ & 65 & 10 & 115 & 10 & \\
\hline Mean \pm SD & $62.7 \pm 13.5$ & & $66.3 \pm 10.1$ & & $<0.001$ \\
\hline Range & $25-91$ & & $34-89$ & & \\
\hline Age at diagnosis, years & & & & & $<0.001$ \\
\hline $19-49$ & 280 & 43 & 245 & 21 & \\
\hline $50-64$ & 249 & 38 & 545 & 46 & \\
\hline $65-86$ & 123 & 19 & 398 & 34 & \\
\hline Mean \pm SD & $51.9 \pm 14.2$ & & $58.8 \pm 10.1$ & & $<0.001$ \\
\hline Range & $19-86$ & & $25-80$ & & \\
\hline Time since diagnosis, years & & & & & $<0.001$ \\
\hline $2-5$ & 151 & 23 & & & \\
\hline $5-10$ & 236 & 36 & 1188 & 100 & \\
\hline $10-20$ & 181 & 28 & & & \\
\hline$\geq 20$ & 84 & 13 & & & \\
\hline Mean \pm SD & $10.8 \pm 7.5$ & & $7.4 \pm 0.9$ & & $<0.001$ \\
\hline Range & $2.5-43.6$ & & $5.3-9.9$ & & \\
\hline Number of comorbidities & & & & & $<0.001$ \\
\hline 0 & 72 & 11 & 270 & 23 & \\
\hline $1-2$ & 251 & 39 & 548 & 47 & \\
\hline $3-5$ & 247 & 38 & 295 & 25 & \\
\hline$\geq 6$ & 76 & 12 & 59 & 5 & \\
\hline Mean \pm SD & $2.9 \pm 2.1$ & & $2.0 \pm 1.9$ & & $<0.001$ \\
\hline Range & $0-11$ & & $0-20$ & & \\
\hline
\end{tabular}

$B C$ breast cancer; $N H L$ non-Hodgkin lymphoma; $S D$ standard deviation.

Tests for differences between the NHL and BC groups were conducted using two-sample t tests, chi-square tests and Fisher exact tests. 
Table 2 Comparison of exploratory factor analyses of IOC items for breast cancer and non-Hodgkin lymphoma survivor samples

Factor loadings from exploratory factor analyses

BC survivors NHL survivors

Altruism/Empathy

Having had cancer has made me more willing to help others

0.86

0.89

Because I had cancer I am more understanding of what other people feel

0.80

0.84

I feel a special bond with people with cancer

0.77

0.83

I feel I should give something back to others

0.76

0.86

I place a higher value on my relationships with family or friends than I did before having had cancer

I learned something about myself because of having had cancer

Health awareness

Having had cancer has made me more concerned about my health

I do not take my body for granted since I had cancer

I am more aware of physical problems or changes

Having had cancer has made me take better care of myself

Meaning of cancer

Because of cancer I have more confidence in myself

Having had cancer has given me direction in life

Because of cancer I have become better about expressing what I want

Because of having had cancer I feel that I have more control of my life

Having had cancer turned into a reason to make changes in my life

Positive self-evaluation

I consider myself to be a cancer survivor

I feel a sense of pride or accomplishment from surviving cancer

I feel that I am a role model

I learned something about myself because of having had cancer

0.71

Positive outlook

Having had cancer has made me realize that time is precious

I learned something about life because of having had cancer

Having had cancer has strengthened my religious faith or sense of spirituality

Appearance concerns

I worry about how my body looks

I feel disfigured

I sometimes wear clothing to cover parts of my body

Body change concerns*

I am bothered that my body cannot do what it could before

I am concerned that my energy has not returned

Having had cancer has made me feel old.

Life interferences*

Having had cancer has made me feel alone

I feel like cancer runs my life

Having had cancer has made me feel like some people do not understand me

I feel guilty today for not having been available to my family

Ongoing symptoms interfere with my life. 
Factor loadings from exploratory factor analyses

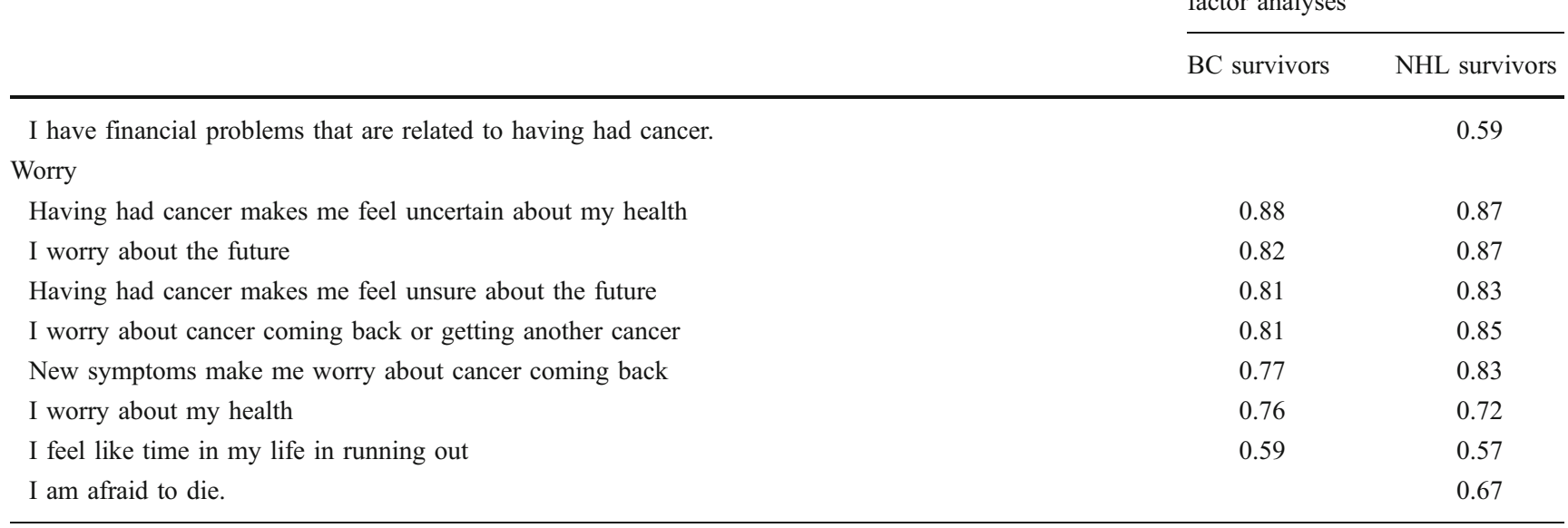

$B C$ breast cancer; NHL non-Hodgkin lymphoma

Body change concerns and life interferences emerged as a single domain in the factor analysis on the NHL sample.

between the IOCv2 negative scales and the PCL-C. These correlations are consistent with the parallel approaches of the PCL-C and IOCv2, which both focus on concerns that respondents attribute specifically to their cancer experience. The IOCv2 negative scales were uncorrelated with the PTGI, and weakly inversely correlated with Social Support.

With regard to the negative subscales, IOCv2 Appearance Concerns showed diffuse connection with various physical and emotional scales. IOCv2 Body Change Concerns was correlated with scales measuring physical concerns, and also correlated with mental and emotional scales; this is consistent with the item content of Body Change Concerns, which focuses on emotional reactions to physical changes. IOCv2 Life Interferences showed correlation with each of the FACTG subscales and SF-36 Mental, and was most strongly correlated with FACT-LYM and PCL-C Total. IOCv2 Worry was most strongly correlated with FACT-G Emotional, which contains items pertaining to worry. IOCv2 Employment Concerns showed correlation with the FACT-G, SF-36 and PCL-C, suggesting more employment concerns among respondents with poorer overall HRQOL. The IOCv2 Relationship Concerns subscales were inversely correlated with the Social Support scales, and also correlated with the FACT-G, FACT-LYM, SF-36 Mental and PCL-C scales, suggesting more relationship concerns among respondents with poorer HRQOL.

\section{Comparison of IOCv2 scores}

Table 6 provides Cronbach's alpha for the IOCv2 scales for both survivor groups. All alphas for the $\mathrm{BC}$ group exceeded 0.75. All alphas for the NHL group exceeded 0.70 with the exception of Relationship Concerns (non-partnered), which had alpha $=0.59$. Many authorities consider alpha $>0.70$ acceptable [48], while others accept alpha $>0.50$ or 0.60 [49].

For both groups, scores on all IOCv2 scales ranged over all or most of the possible 1 to 5 range, suggesting good potential for sensitivity and discrimination.

Table 6 compares unadjusted and adjusted mean IOCv2 scores for the BC and NHL survivors, with the adjustment accounting for differences in age and years since diagnosis. To account for gender differences, adjusted means for the BC sample and women in the NHL sample are also compared.

Unadjusted and adjusted mean scores for both groups on the Positive Impact Summary Scale were in the "agree" range, indicating endorsement of positive impacts due to cancer in both groups. A marginally significant difference in unadjusted mean scores between the $\mathrm{BC}$ and $\mathrm{NHL}$ survivors became non-significant after adjusting for age and time since diagnosis. However, there were differences between the groups on positive subscales that remained after adjustment. In particular, the NHL survivors had higher mean scores on Altruism/Empathy and lower scores on Meaning of Cancer. Differences on the latter were partially accounted for by gender differences, with NHL men scoring lower on Meaning of Cancer than NHL women on average.

Mean scores on the Negative Impact Summary Scale were in the "disagree" range and differed between the groups in adjusted and unadjusted analyses, with the $\mathrm{BC}$ survivors reporting more negative impacts. Mean scores on Appearance Concerns were a full point higher among the $\mathrm{BC}$ survivors. Differences on Body Change Concerns, Life Interferences and Worry were non-significant after adjustment.

The BC survivors reported higher levels of relationship concerns, both for partnered and unpartnered respondents, with these differences remaining significant after adjustment. 
Table 3 Results of reproducibility analysis comparing factor structure for non-Hodgkin lymphoma and breast cancer survivor samples

IOCv2 subscales and items

Altruism/Empathy

Having had cancer has made me more willing to help others

Because I had cancer I am more understanding of what others feel

I feel a special bond with people with cancer

I feel I should give something back to others

Health awareness

Having had cancer has made me more concerned about my health.

I do not take my body for granted since I had cancer.

I am more aware of physical problems or changes.

Having had cancer has made me take better care of myself.

Meaning of cancer

Because of cancer I have more confidence in myself

Having had cancer has given me direction in life

Because of cancer I have become better about expressing what I want

Because of having had cancer I feel that I have more control of my life

Having had cancer turned into a reason to make changes in my life

Positive self-evaluation

I feel a sense of pride or accomplishment from surviving cancer

I consider myself to be a cancer survivor

I learned something about myself because of having had cancer

I feel that I am a role model

Appearance concerns

I feel disfigured

I sometimes wear clothing to cover parts of my body

I worry about how my body looks

Body change concerns

I am bothered that my body cannot do what it could before

I am concerned that my energy has not returned

Having had cancer has made me feel old.

Life interferences

Uncertainty about my future affects my decisions to make plans

Having had cancer has made me feel alone.

Having had cancer keeps me from doing activities I enjoy

I feel like cancer runs my life.

Having had cancer has made me feel that some people do not understand me

I feel guilty today for not having been available to my family

Ongoing symptoms interfere with my life.

Worry

Having had cancer makes me feel uncertain about my health

I worry about the future

Having had cancer makes me feel unsure about the future

I worry about cancer coming back

New symptoms make me worry about cancer coming back

I worry about my health

I feel like time in my life in running out
Factor Congruence

Item Congruence

0.96

0.96

0.98

0.98

0.95

1.00

0.94

0.99

0.96

0.98

0.99

0.99

0.99

0.98

0.97

0.97

0.96

0.98

0.96

0.98

0.98

0.97

0.99

0.99

0.99

0.88

0.34

0.98

0.58

0.99

0.95

0.90

0.95

0.98

0.98

0.97

0.97

0.95

0.97

0.97

0.93 
Table 4 FACT, PCL-C, SF-36, PTGI, and Social Support scores in the NHL survivor sample and correlations $(r)$ with IOCv2 scores on positive scales

\begin{tabular}{|c|c|c|c|c|c|c|}
\hline \multirow[t]{2}{*}{ Instrument $^{\mathrm{a}}$} & \multirow{2}{*}{$\begin{array}{l}\text { Mean } \\
\pm \text { SD }\end{array}$} & \multirow{2}{*}{$\begin{array}{l}\text { Positive impact } \\
\text { summary score }\end{array}$} & \multicolumn{4}{|l|}{ Positive subscales } \\
\hline & & & Altruism/ Empathy & Health awareness & Meaning of cancer & Positive self-evaluation \\
\hline \multicolumn{7}{|l|}{ FACT-G (+) } \\
\hline Total & $88 \pm 15$ & 0.08 & 0.04 & -0.02 & 0.07 & 0.15 \\
\hline Physical & $24 \pm 5$ & -0.07 & -0.09 & -0.08 & -0.06 & 0.01 \\
\hline Social/Family & $23 \pm 5$ & 0.19 & 0.21 & 0.07 & 0.13 & 0.20 \\
\hline Emotional & $20 \pm 4$ & 0.01 & -0.03 & -0.09 & 0.02 & 0.13 \\
\hline Functional & $21 \pm 6$ & 0.13 & 0.07 & 0.02 & 0.14 & 0.17 \\
\hline LYM & $137 \pm 22$ & 0.02 & -0.01 & -0.06 & 0.01 & 0.11 \\
\hline \multicolumn{7}{|l|}{ SF-36 (+) } \\
\hline Physical comp. & $46 \pm 10$ & -0.01 & -0.06 & -0.01 & 0.01 & 0.03 \\
\hline Mental comp. & $50 \pm 11$ & -0.00 & -0.03 & -0.06 & 0.01 & 0.08 \\
\hline \multicolumn{7}{|l|}{ SF-36 subscales } \\
\hline Physical funct. & $45 \pm 12$ & -0.02 & -0.06 & 0.01 & -0.03 & 0.01 \\
\hline Role-physical & $45 \pm 12$ & -0.01 & -0.08 & -0.03 & 0.02 & 0.03 \\
\hline Bodily pain & $51 \pm 10$ & -0.02 & -0.07 & -0.02 & -0.00 & 0.02 \\
\hline General health & $46 \pm 11$ & 0.02 & -0.04 & -0.05 & 0.05 & 0.08 \\
\hline Vitality & $49 \pm 12$ & 0.06 & -0.01 & -0.01 & 0.08 & 0.09 \\
\hline Social function. & $49 \pm 11$ & 0.00 & -0.04 & -0.04 & 0.02 & 0.06 \\
\hline Role-emotional & $46 \pm 13$ & -0.04 & -0.07 & -0.05 & -0.04 & 0.04 \\
\hline Mental health & $50 \pm 10$ & -0.03 & -0.05 & -0.07 & -0.02 & 0.05 \\
\hline \multicolumn{7}{|l|}{ PCL-C (-) } \\
\hline Total & $26 \pm 9$ & 0.07 & 0.08 & 0.12 & 0.05 & -0.03 \\
\hline Re-experiencing & $7 \pm 3$ & 0.14 & 0.11 & 0.17 & 0.12 & 0.04 \\
\hline Avoidance & $10 \pm 4$ & -0.01 & 0.01 & 0.09 & -0.02 & -0.11 \\
\hline Arousal & $9 \pm 4$ & 0.09 & 0.11 & 0.08 & 0.06 & 0.03 \\
\hline \multicolumn{7}{|l|}{ PTGI (+) } \\
\hline Total & $62 \pm 24$ & $0.75^{* * *}$ & $0.64 * * *$ & $0.45 * *$ & $0.69 * * *$ & $0.56 * *$ \\
\hline Rel'ship w/others & $23 \pm 9$ & $0.67 * * *$ & $0.62 * * *$ & $0.38 *$ & $0.58 * *$ & $0.53 * *$ \\
\hline New possibilities & $11 \pm 7$ & $0.71 * * *$ & $0.56^{* *}$ & $0.41 *$ & $0.70^{* * *}$ & $0.50 * *$ \\
\hline Pers'l strength & $12 \pm 5$ & $0.67 * * *$ & $0.55^{* *}$ & $0.38 *$ & $0.65^{* * *}$ & $0.51 * *$ \\
\hline Spiritual change & $6 \pm 3$ & $0.60 * * *$ & $0.55^{* *}$ & $0.34^{*}$ & $0.53 * *$ & $0.49 * *$ \\
\hline Apprec. of life & $10 \pm 4$ & $0.68 * * *$ & $0.56^{* *}$ & $0.51^{* *}$ & $0.59 * *$ & $0.47 * *$ \\
\hline \multicolumn{7}{|l|}{ Social support $(+)$} \\
\hline Total & $84 \pm 16$ & 0.10 & 0.09 & 0.02 & 0.07 & 0.14 \\
\hline Emotional/Info & $81 \pm 18$ & 0.09 & 0.07 & 0.01 & 0.07 & 0.12 \\
\hline Tangible & $84 \pm 19$ & 0.08 & 0.08 & 0.02 & 0.04 & 0.13 \\
\hline Affectionate & $90 \pm 17$ & 0.09 & 0.10 & 0.02 & 0.05 & 0.10 \\
\hline Pos. interaction & $86 \pm 19$ & 0.09 & 0.08 & 0.03 & 0.07 & 0.11 \\
\hline
\end{tabular}

FACT Functional Assessment of Cancer Therapy; PCL-C Post-traumatic Stress Disorder Checklist-Civilian Version; PTGI Post-Traumatic Growth Inventory.

$* 0.30 \leq|r|<0.45 ; * * 0.45 \leq|r|<0.60 ; * * *|r| \geq 0.60$; and $P<0.005$ for test that correlation equals zero.

${ }^{\text {a }}$ Instruments completed concurrently with the IOC. Direction of scoring is indicated in parentheses, with a negative (-) sign indicating that higher scores correspond to poorer functioning and a positive $(+)$ sign indicating that higher scores correspond to better functioning. 
Table 5 Correlations $(r)$ of FACT, PCL-C, SF-36, PTGI, and Social Support scores in the NHL survivor sample with IOCv2 scores on negative scales

\begin{tabular}{|c|c|c|c|c|c|c|c|c|}
\hline \multirow[t]{2}{*}{ Instrument $^{\mathrm{a}}$} & \multirow{2}{*}{$\begin{array}{l}\text { Negative impact } \\
\text { summary score }\end{array}$} & \multicolumn{4}{|c|}{ Negative subscales } & \multirow{2}{*}{$\begin{array}{l}\text { Employ } \\
\text { conc }\end{array}$} & \multirow{2}{*}{$\begin{array}{l}\text { Rel'ship conc: } \\
\text { not partnered }\end{array}$} & \multirow{2}{*}{$\begin{array}{l}\text { Rel'ship conc: } \\
\text { partnered }\end{array}$} \\
\hline & & $\begin{array}{l}\text { Appear } \\
\text { conc }\end{array}$ & $\begin{array}{l}\text { Body chg } \\
\text { conc }\end{array}$ & $\begin{array}{l}\text { Life } \\
\text { interfer }\end{array}$ & Worry & & & \\
\hline \multicolumn{9}{|l|}{ FACT-G $(+)$} \\
\hline Total & $-0.68 * * *$ & $-0.45 * *$ & $-0.60 * * *$ & $-0.59 * *$ & $-0.52 * *$ & $-0.45^{* *}$ & $-0.64 * * *$ & $-0.45 * *$ \\
\hline Physical & $-0.59 * *$ & $-0.38 *$ & $-0.65 * * *$ & $-0.52 * *$ & $-0.38 *$ & $-0.36^{*}$ & $-0.55^{* *}$ & -0.28 \\
\hline Social/Family & $-0.39 *$ & $-0.30^{*}$ & -0.27 & $-0.31 *$ & $-0.34^{*}$ & $-0.33^{*}$ & $-0.42 *$ & $-0.49 * *$ \\
\hline Emotional & $-0.70 * * *$ & $-0.40^{*}$ & $-0.50 * *$ & $-0.59 * *$ & $-0.63 * * *$ & $-0.38^{*}$ & $-0.62 * * *$ & $-0.36^{*}$ \\
\hline Functional & $-0.53 * *$ & $-0.37^{*}$ & $-0.51 * *$ & $-0.47 * *$ & $-0.38 *$ & $-0.37^{*}$ & $-0.45^{* *}$ & $-0.32 *$ \\
\hline LYM & $-0.73 * * *$ & $-0.47 * *$ & $-0.64 * * *$ & $-0.63 * * *$ & $-0.56^{* *}$ & $-0.48 * *$ & $-0.62 * * *$ & $-0.43^{*}$ \\
\hline \multicolumn{9}{|l|}{ SF-36 $(+)$} \\
\hline Physical comp. & $-0.30 *$ & -0.19 & $-0.41^{*}$ & -0.29 & -0.15 & -0.13 & -0.04 & -0.13 \\
\hline Mental comp. & $-0.53 * *$ & $-0.34 *$ & $-0.47 * *$ & $-0.47 * *$ & $-0.41^{*}$ & $-0.34 *$ & $-0.54 * *$ & $-0.31^{*}$ \\
\hline \multicolumn{9}{|l|}{ SF-36 Subscales } \\
\hline Physical funct. & -0.26 & -0.15 & $-0.38^{*}$ & -0.28 & -0.10 & -0.12 & -0.00 & -0.13 \\
\hline Role-physical & $-0.35^{*}$ & -0.22 & $-0.46^{* *}$ & $-0.35^{*}$ & -0.17 & -0.16 & -0.19 & -0.15 \\
\hline Bodily pain & $-0.35^{*}$ & -0.22 & $-0.37^{*}$ & $-0.33^{*}$ & -0.22 & -0.17 & -0.19 & -0.18 \\
\hline General health & $-0.52 * *$ & $-0.32 *$ & $-0.50 * *$ & $-0.44 *$ & $-0.41^{*}$ & $-0.31^{*}$ & $-0.32 *$ & -0.23 \\
\hline Vitality & $-0.50 * *$ & $-0.33^{*}$ & $-0.55 * *$ & $-0.40^{*}$ & $-0.36^{*}$ & -0.28 & $-0.37^{*}$ & -0.27 \\
\hline Social function. & $-0.44 *$ & $-0.30^{*}$ & $-0.42 *$ & $-0.45^{* *}$ & -0.28 & -0.27 & $-0.34 *$ & -0.29 \\
\hline Role-emotional & $-0.41 *$ & -0.25 & $-0.45^{* *}$ & $-0.41 *$ & -0.25 & -0.24 & $-0.36^{*}$ & -0.22 \\
\hline Mental health & $-0.53 * *$ & $-0.32^{*}$ & $-0.44 *$ & $-0.46^{* *}$ & $-0.44^{*}$ & $-0.36^{*}$ & $-0.49 * *$ & $-0.32 *$ \\
\hline \multicolumn{9}{|l|}{ PCL-C (-) } \\
\hline Total & $0.70 * * *$ & $0.41^{*}$ & $0.56 * *$ & $0.63 * * *$ & $0.57 * *$ & $0.43 *$ & $0.59 * *$ & $0.39 *$ \\
\hline Re-experiencing & $0.54 * *$ & 0.29 & $0.40^{*}$ & $0.51 * *$ & $0.47 * *$ & $0.37 *$ & $0.53 * *$ & 0.22 \\
\hline Avoidance & $0.66^{* * *}$ & $0.39 *$ & $0.52 * *$ & $0.61 * * *$ & $0.54 * *$ & $0.36^{*}$ & $0.63 * * *$ & $0.42 *$ \\
\hline Arousal & $0.59 * *$ & $0.37 *$ & $0.51 * *$ & $0.52 * *$ & $0.47 * *$ & $0.37^{*}$ & $0.39 *$ & $0.33^{*}$ \\
\hline \multicolumn{9}{|l|}{ PTGI (+) } \\
\hline Total & 0.09 & 0.07 & 0.11 & 0.15 & 0.00 & 0.05 & 0.03 & -0.10 \\
\hline Rel'ship w/others & 0.07 & 0.04 & 0.12 & 0.13 & -0.01 & 0.01 & -0.03 & -0.13 \\
\hline New possibilities & 0.06 & 0.09 & 0.06 & 0.13 & -0.02 & 0.09 & 0.08 & -0.05 \\
\hline Pers'l strength & 0.06 & 0.05 & 0.08 & 0.12 & -0.02 & 0.04 & -0.00 & -0.08 \\
\hline Spiritual change & 0.10 & 0.08 & 0.12 & 0.17 & -0.01 & 0.06 & 0.04 & -0.05 \\
\hline Apprec. of life & 0.17 & 0.08 & 0.14 & 0.19 & 0.12 & 0.10 & 0.16 & -0.11 \\
\hline \multicolumn{9}{|l|}{ Social Support (+) } \\
\hline Total & -0.29 & -0.25 & -0.18 & -0.26 & -0.24 & -0.29 & $-0.43 *$ & $-0.41^{*}$ \\
\hline Emotional/Info & -0.28 & -0.18 & -0.18 & -0.27 & -0.23 & -0.26 & $-0.42 *$ & $-0.34^{*}$ \\
\hline Tangible & -0.20 & -0.19 & -0.11 & -0.18 & -0.17 & -0.20 & $-0.31^{*}$ & $-0.30^{*}$ \\
\hline Affectionate & -0.20 & -0.23 & -0.10 & -0.15 & -0.17 & -0.25 & -0.28 & $-0.41^{*}$ \\
\hline Pos. interaction & -0.26 & -0.29 & -.18 & -0.22 & -0.19 & -0.28 & $-0.38 *$ & $-0.41^{*}$ \\
\hline
\end{tabular}

conc concerns; FACT Functional Assessment of Cancer Therapy; PCL-C Post-traumatic Stress Disorder Checklist-Civilian Version; PTGI PostTraumatic Growth Inventory.

$* 0.30 \leq|r|<0.45 ; * * 0.45 \leq|r|<0.60 ; * * *|r| \geq 0.60$; and $P<0.005$ for test that correlation equals zero.

${ }^{a}$ Instruments completed concurrently with the IOC. Direction of scoring is indicated in parentheses, with a negative (-) sign indicating that higher scores correspond to poorer functioning and a positive $(+)$ sign indicating that higher scores correspond to better functioning. 
Table 6 Cronbach's alpha statistics and unadjusted and adjusted mean IOCv2 scale scores for non-Hodgkin lymphoma and breast cancer survivor samples

\begin{tabular}{|c|c|c|c|c|c|c|c|c|c|c|}
\hline \multirow[t]{2}{*}{ Scale } & \multicolumn{2}{|c|}{$\begin{array}{l}\text { Cronbach's } \\
\text { alpha }\end{array}$} & \multicolumn{3}{|c|}{ Unadjusted means \pm SD (range) } & \multicolumn{5}{|c|}{ Adjusted means } \\
\hline & $\mathrm{BC}$ & NHL & $\mathrm{BC}$ & NHL & $\begin{array}{l}\text { P, NHL } \\
\text { v BC }\end{array}$ & $\mathrm{BC}$ & $\begin{array}{l}\text { NHL, } \\
\text { women only }\end{array}$ & $\begin{array}{l}\text { NHL, men } \\
\text { and women }\end{array}$ & $\begin{array}{l}\mathrm{P}, \mathrm{BC} \text { v NHL } \\
\text { women only }\end{array}$ & $\begin{array}{l}\mathrm{P}, \mathrm{BC} v \mathrm{NHL} \\
\text { men and women }\end{array}$ \\
\hline \multicolumn{11}{|l|}{ Higher-order scales } \\
\hline Pos summary & 0.79 & 0.90 & $3.6 \pm 0.6(1.2-5)$ & $3.5 \pm 0.8(1-5)$ & 0.04 & 3.6 & 3.7 & 3.5 & 0.22 & 0.09 \\
\hline Neg summary & 0.81 & 0.91 & $2.4 \pm 0.7(1-4.8)$ & $2.1 \pm 0.7(1-4.7)$ & $<0.001$ & 2.4 & 2.2 & 2.2 & 0.007 & $<0.001$ \\
\hline \multicolumn{11}{|l|}{ Pos. subscales } \\
\hline Altru/Empathy & 0.82 & 0.87 & $3.8 \pm 0.7(1-5)$ & $3.9 \pm 0.9(1-5)$ & $<0.001$ & 3.7 & 4.1 & 3.9 & $<0.001$ & 0.004 \\
\hline Health aware & 0.80 & 0.71 & $3.8 \pm 0.7(1-5)$ & $3.7 \pm 0.8(1-5)$ & 0.01 & 3.8 & 3.8 & 3.7 & 0.94 & 0.26 \\
\hline Meaning of $\mathrm{Ca}$ & 0.87 & 0.87 & $3.0 \pm 0.8(1-5)$ & $2.8 \pm 1.1(1-5)$ & $<0.001$ & 3.0 & 2.9 & 2.8 & 0.08 & $<0.001$ \\
\hline Pos self-eval & 0.79 & 0.77 & $4.0 \pm 0.7(1-5)$ & $4.0 \pm 0.9(1-5)$ & 0.86 & 4.0 & 4.1 & 3.9 & 0.14 & 0.20 \\
\hline \multicolumn{11}{|l|}{ Neg. subscales } \\
\hline Appear. conc & 0.78 & 0.76 & $2.7 \pm 1.0(1-5)$ & $1.6 \pm 0.8(1-5)$ & $<0.001$ & 2.7 & 1.8 & 1.7 & $<0.001$ & $<0.001$ \\
\hline Body chg conc & 0.82 & 0.83 & $2.6 \pm 1.0(1-5)$ & $2.3 \pm 1.2(1-5)$ & $<0.001$ & 2.5 & 2.5 & 2.5 & 0.66 & 0.54 \\
\hline Life interfer & 0.81 & 0.71 & $1.9 \pm 0.6(1-4.4)$ & $1.9 \pm 0.7(1-4.6)$ & 0.90 & 1.9 & 1.9 & 2.0 & 0.46 & 0.17 \\
\hline Worry & 0.89 & 0.89 & $2.8 \pm 0.8(1-5)$ & $2.5 \pm 1.0(1-5)$ & $<0.001$ & 2.7 & 2.6 & 2.6 & 0.17 & 0.09 \\
\hline \multicolumn{11}{|l|}{ Other subscales } \\
\hline Employ conc & 0.76 & 0.75 & $2.7 \pm 1.1(1-5)$ & $2.4 \pm 1.2(1-5)$ & 0.002 & 2.7 & 2.4 & 2.5 & 0.12 & 0.27 \\
\hline $\begin{array}{l}\text { Rel'ship conc } \\
\text { (not partnered) }\end{array}$ & 0.78 & 0.59 & $2.1 \pm 0.9(1-5)$ & $1.6 \pm 0.9(1-5)$ & $<0.001$ & 2.0 & 1.5 & 1.7 & 0.004 & 0.03 \\
\hline $\begin{array}{l}\text { Rel'ship conc } \\
\text { (partnered) }\end{array}$ & 0.80 & 0.77 & $1.7 \pm 0.6(1-4.5)$ & $1.4 \pm 0.6(1-3.5)$ & $<0.001$ & 1.6 & 1.5 & 1.4 & 0.003 & $<0.001$ \\
\hline
\end{tabular}

$B C$ breast cancer; $N H L$ non-Hodgkin lymphoma.

For all scales, potential scores range from 1 to 5 , with higher scores indicating stronger endorsement of the content area and with a score of 3 as neutral. Adjusted means are adjusted for age and years since diagnosis.

\section{Discussion}

There is a need for well-validated measures of the cancerspecific HRQOL of long-term cancer survivors that can be used across the spectrum of cancer diagnoses. Our study was undertaken to examine the generalizability of the IOCv2 across two distinct survivor groups. We compared the measurement of the impact of cancer in large samples of BC survivors and NHL survivors and found evidence for similar impact domains across the two groups, providing confidence that the IOCv2 scales measure common and important survivor concerns.

Compared to the $\mathrm{BC}$ group, the NHL survivors represented a broader range of time since diagnosis and ages at diagnosis, as well as both genders, and came from a different geographic area of the US. The good psychometric properties of the scales in this distinct group, as well as the participation of diverse samples of survivors in earlier stages of IOC development, support the appropriateness of the IOCv2 as a tool to measure the psychosocial impacts of cancer in the broader long-term survivor population. However, some limitations in generalizability remain. While our NHL survivor sample included 151 participants who were survivors within $2-5$ years of diagnosis, more work is needed to confirm that the IOCv2 items and factor structure capture domains that are relevant to early survivors, who are closer to the transition from patient to survivor and thus may be experiencing different psychosocial needs [7].

Some differences emerged in the independent scalings in the BC and NHL samples. Content of the Life Interferences domain differed somewhat across samples, and merged with Body Change Concerns in NHL survivors. Consistent with this is the high correlation between these two subscales in the $\mathrm{BC}$ sample $(r=0.6)$ [30]. Overall these findings suggest overlap between these two domains. While the content of the Life Interferences subscale was not fully congruent between the $\mathrm{BC}$ and NHL survivors, the importance of a life interferences domain for NHL survivors is supported by the study of Bellizzi et al. [16], who report substantial proportions of NHL survivors endorsing negative impacts on ability to participate in social and exercise activities and to enjoy life as well as on financial, career and insurance situations.

The Positive Outlook subscale, which emerged in early IOC scaling with 193 survivors of breast, colorectal, 
lymphatic and prostate cancers, also emerged in the NHL sample but was not evident in the large $\mathrm{BC}$ sample. The sample used for early IOC scaling included 49 (25\%) lymphoma (Hodgkin and NHL) survivors, suggesting that this domain might reflect concerns of this group that are not as prevalent in $\mathrm{BC}$ survivors. Alternatively, the difference may be due to the restriction of the $\mathrm{BC}$ sample to long-term survivors who were within 10 years of their diagnosis; one of the theoretical foundations of growth is sufficient time to think about and integrate experiences into one's life [50], and the time interval may have been insufficient for some of the BC survivors. Future research may explore the universality of this construct and its association with time since diagnosis.

We have also compared IOCv2 scores in NHL and BC survivors. Normative and comparative scores across groups will be important in facilitating interpretation and identifying at-risk populations to target for intervention. Differences between the NHL and BC groups for several domains, including the Negative Impact Summary scale and Altruism/ Empathy, Meaning of Cancer, Appearance Concerns and Relationship Concerns subscales, suggest that the IOCv2 scales are able to distinguish differential impacts in different survivor groups. While it is beyond the scope of this paper to fully explore the antecedents of these differences, we can offer several observations. Subscale comparisons suggest that the difference in Negative Impact Summary scores between the $\mathrm{BC}$ and NHL groups may be largely attributable to differences on the Appearance Concerns subscale, with BC survivors scoring about a full point higher on this domain. Body image is a widely reported concern among BC survivors (e.g. [51, 52]). However, this should not be taken as evidence that appearance concerns are exclusive to BC survivors, as such concerns are also reported among survivors with other diagnoses (e.g. [53-56]). Among perceived positive impacts of cancer, the NHL survivors scored higher on Altruism/ Empathy while the BC survivors scored higher on Meaning of Cancer. This may be interpreted as a difference in benefitfinding domains in these two groups. There is a sizable literature examining predictors of posttraumatic growth in cancer survivors; within this literature, disease and treatment characteristics have shown inconsistent associations with posttraumatic growth [57]. To the extent that the IOCv2 identifies four distinct positive impact domains which may be differentially associated with disease and treatment characteristics, it may assist in providing greater differentiation and clarity in this area in future research. We plan future work to further characterize the perceived impact of cancer in NHL survivors using the IOCv2 scales.

The higher order positive and negative summary scores of the IOCv2 provide aggregate measures of impacts that may be especially useful when data reduction is important. However, as we saw with the positive scale scores in $\mathrm{BC}$ and NHL survivors, groups can differ on subscales while having little or no difference in summary scores. Hence we would recommend that researchers using the IOCv2 examine both summary and subscale scores. Indeed, the capability of eliciting different patterns of scores across different survivor groups is an advantage of a multidimensional measure such as the IOCv2.

Our study has several limitations. Respondents tended to be older and were less racially/ethnically diverse than nonrespondents in both groups, creating the potential for response biases. Since our surveys were cross-sectional, we were not able to evaluate test-retest reliability nor the ability of the IOCv2 to measure change over time, which will be important to assess in future research. In addition, the 47 items for the IOCv2 were selected based on data collected via full administration of the 81 -item IOC questionnaire. It will be important to examine whether the domain and factor structure of the IOCv2 holds up when the 47-item only instrument is utilized in future studies. Work is also needed to evaluate the IOCv2 in clinical settings, where it could be used as a screening tool or to measure outcomes in a clinical trial or program evaluation.

Acknowledgements Funding for this research was provided by the National Cancer Institute (CA016042 and CA101492), an American Cancer Clinical Research Professorship (Dr. Ganz), the American Cancer Society (DSW-0321301-SW), AHRQ NRSA Training Grant (T32-HS000032) and the University of North Carolina Research Council.

Open Access This article is distributed under the terms of the Creative Commons Attribution Noncommercial License which permits any noncommercial use, distribution, and reproduction in any medium, provided the original author(s) and source are credited.

\section{References}

1. Ries LAG, Melbert D, Krapcho $M$ et al (eds). SEER Cancer Statistics Review, 1975-2005, National Cancer Institute. Bethesda, MD, http://seer.cancer.gov/csr/1975_2005/, based on November 2007 SEER data submission, posted to the SEER web site, accessed March 2009.

2. Gotay CC, Muraoka MY. Quality of life in long-term survivors of adult-onset cancers. J Natl Cancer Inst. 1998;90:656-67.

3. Bloom JR, Petersen DM, Kang SH. Multi-dimensional quality of life among long-term $(5+$ years $)$ adult cancer survivors. Psychooncology. 2007;16:691-706.

4. Alfano CM, Rowland JH. Recovery issues in cancer survivorship: a new challenge for supportive care. Cancer J. 2006;12:432-43.

5. Aziz NM. Late effects of cancer treatment. In: Chang AE, Ganz PA, Hayes DF, et al., editors. Oncology: an evidence-based approach. New York: Springer; 2006. p. 1768-90.

6. Aziz NM. Cancer survivorship research: state of knowledge, challenges and opportunities. Acta Oncologica. 2007;46:417-32.

7. Hewitt M, Greenfield S, Stovall E (eds). From cancer patient to cancer survivor: lost in transition. The National Academies Press, 2006. 
8. Park SY, Bae DS, Nam JH, Park CT, Cho CH, Lee JM, et al. Quality of life and sexual problems in disease-free survivors of cervical cancer compared with the general population. Cancer. 2007;110(12):2716-25.

9. Noorda EM, van Kreij RHJ, Vrouenraets BC, Nieweg OE, Muller M, Kroon BBR, et al. The health-related quality of life of longterm survivors of melanoma treated with isolated limb perfusion. Eur J Surg Oncol. 2007;33(6):776-82.

10. Hodgkinson K, Butow P, Fuchs A, Hunt GE, Stenlake A, Hobbs $\mathrm{KM}$, et al. Long-term survival from gynecologic cancer: psychosocial outcomes, supportive care needs and positive outcomes. Gynecologic Oncol. 2007;104(2):381-9.

11. Weaver KE, Llabre MM, Lechner SC, Penedo F, Antoni MH. Comparing unidimensional and multidimensional models of benefit finding in breast and prostate cancer. Qual Life Res. 2008; $17: 771-81$.

12. Mols F, Vingerhoets AJJM, Coebergh JWW, van de Poll-Franse LV. Well-being, postraumatic growth and benefit finding in long-term breast cancer survivors. Psychology \& Health. 2009;24:583-95.

13. Porter LS, Clayton MF, Belyea M, Mishel M, Gil KM, Germino BB. Predicting negative mood state and personal growth in African American and white long-term breast cancer survivors. Ann Behav Med. 2006;31:195-204.

14. Foley KL, Farmer DF, Petronis VM, Smith RG, McGraw S, Smith $\mathrm{K}$, et al. A qualitative exploration of the cancer experience among long-term survivors: comparisons by cancer type, ethnicity, gender, and age. Psychooncology. 2006;15(3):248-58.

15. Bower JE, Meyerowitz BE, Desmond KA, Bernaards CA, Rowland JH, Ganz PA. Perceptions of positive meaning and vulnerability following breast cancer: predictors and outcomes among long-term breast cancer survivors. Ann Behav Med. 2005;29:236-45.

16. Bellizzi KM, Miller MF, Arora NK, Rowland JH. Positive and negative life changes experienced by survivors of non-Hodgkin's lymphoma. Ann Behav Med. 2007;34:188-99.

17. Ferrell BR, Hassey Dow K, Grant M. Measurement of the quality of life in cancer survivors. Qual Life Res. 1995;4:523-31.

18. Wyatt GKH, Friedman LL. Development and testing of a quality of life model for long-term female cancer survivors. Qual Life Res. 1996;5:387-94.

19. Avis NE, Smith KW, McGraw S, et al. Assessing quality of life in adult cancer survivors (QLACS). Qual Life Res. 2005;14:1007-23.

20. Avis NE, Ip E, Foley KL. Evaluation of the Quality of Life in Adult Cancer Survivors (QLACS) scale for long-term cancer survivors in a sample of breast cancer survivors. Health Qual Life Outcomes. 2006;4:92-102.

21. Ferrell BR, Hassey Dow K, Grant M. Measurement of the quality of life in cancer survivors. Qual Life Res. 1995;4:523-31.

22. Avis NE, Smith KW, McGraw S, Smith RG, Petronis VM, Carver CS. Assessing quality of life in adult cancer survivors (QLACS). Qual Life Res. 2005;14:1007-23.

23. Avis NE, Ip E, Foley KL. Evaluation of the Quality of Life in Adult Cancer Survivors (QLACS) scale for long-term cancer survivors in a sample of breast cancer survivors. Health Qual Life Outcomes. 2006;4:92-102.

24. Ganz PA, Day R, Ware JE Jr, Redmond C, Fisher B. Base-line quality-of-life assessment in the national surgical adjuvant breast and bowel project breast cancer prevention trial. J Natl Cancer Inst. 1995;87:1372-82.

25. Stanton AL, Bernaards CA, Ganz PA. The BCPT symptom scales: a measure of physical symptoms for women diagnosed with or at risk for breast cancer. J Natl Cancer Inst. 2005;97:448-56.

26. Lee-Jones C, Humphris G, Dixon R, Hatcher MB. Fear of cancer recurrence - a literature review and proposed cognitive formulation to explain exacerbation of recurrence fears. Psychooncology. 1997;6:95-105.
27. Gotay CC, Pagano IS. Assessment of survivor concerns (ASC): a newly proposed brief questionnaire. Health Qual Life Outcomes. 2007;5:15-25.

28. Zebrack BJ, Ganz PA, Bernaards CA, et al. Assessing the impact of cancer: development of a new instrument for long-term survivors. Psychooncol. 2006;15:407-21.

29. Zebrack BJ, Yi J, Petersen L, Ganz PA. The impact of cancer and quality of life for long-term survivors. Psychooncol. 2008;17:891900.

30. Crespi CM, Ganz PA, Petersen L, et al. Refinement of the Impact of Cancer (IOC) scale in a sample of long-term breast cancer survivors. J Natl Cancer Inst. 2008;100:1530-41.

31. Bower JE, Meyerowitz BE, Desmond KA, et al. Perceptions of positive meaning and vulnerability following breast cancer: predictors and outcomes among long-term breast cancer survivors. Ann Behav Med. 2005;29:236-45.

32. Bellizzi KM, Miller MF, Arora NK, et al. Positive and negative life changes experienced by survivors of non-Hodgkin's lymphoma. Ann Behav Med. 2007;34:188-99.

33. Hodgkinson K, Butow P, Fuchs A, et al. Long-term survival from gynecologic cancer: psychosocial outcomes, supportive care needs and positive outcomes. Gynecologic Oncol. 2007;104:381-9.

34. Smith SK, Zimmerman S, Williams CS, et al. Post-traumatic stress outcomes in non-Hodgkin's lymphoma survivors. J Clin Oncol. 2008;26:934-41.

35. Caan B, Sternfeld B, Gunderson E, et al. Life After Cancer Epidemiology (LACE) Study: a cohort of early stage breast cancer survivors (United States). Cancer Causes Control. 2005;16:54556.

36. Cella DF, Tulsky DS, Gray G, et al. The functional assessment of cancer therapy scale: development and validation of the general measure. J Clin Oncol. 1993;11:570-9.

37. Cella D, Webster K, Cashy J, et al. Development of a measure of health-related quality of life for non-Hodgkin's lymphoma clinical research: The functional assessment of cancer therapy-Lymphoma (FACT-Lym). Blood. 2005;106:222A. abstract.

38. Ware JE. 36-item short-form health survey (SF-36). 1. Conceptual framework and item selection. Medical Care. 1992;30:473-83.

39. American Psychiatric Association. Diagnostic and statistical manual. 4th ed. Washington: American Psychiatric Association; 1994.

40. Weathers FW, Litz B, Herman D, et al. The PTSD Checklist (PCL-C): Reliability, validity and diagnostic utility. Presented at the 9th Annual Meeting of the International Society for Traumatic Stress Studies, San Antonio, TX, October 24-27, 1993 (abstract).

41. Tedeschi RG, Calhoun LG. The posttraumatic growth inventory: measuring the positive legacy of trauma. J Traum Stress. 1996;9:455-71.

42. Taku K, Cann A, Calhoun LG, et al. The factor structure of the posttraumatic growth inventory: a comparison of five models using confirmatory factor analysis. J Traum Stress. 2008;21:15864.

43. Sherbourne CD, Stewart AL. The MOS social support survey. Soc Sci Med. 1991;32:705-14.

44. McCrae RR, Zonderman AB, Costa PT, et al. Evaluating replicability of factors in the revised NEO personality inventory: confirmatory factor analysis versus procrustes rotation. J Pers Soc Psychol. 1996;70:552-66.

45. Kaiser HF, Hunka S, Bianchini JC. Relating factors between studies based upon different individuals. Multivariate Behav Res. 1971;6:409-22.

46. Burnand B, Kernan WN, Feinstein AR. Indexes and boundaries for 'quantitative significance' in statistical decisions. J Clin Epidemiol. 1990;43:1273-84.

47. Cronbach LJ. Coefficient alpha and the internal structure of test. Psychometrika. 1951;16:297-334. 
48. Nunnally JC, Bernstein IH. Psychometric theory. 3rd ed. New York: McGraw-Hill; 1994.

49. Helmstadter GC. Principles of psychological measurement. New York: Appleton-Century-Crofts; 1964.

50. Tedeschi RG, Park CL, Calhoun LG. Posttraumatic growth: positive changes in the aftermath of crisis. Mahwah: Lawrence Erlbaum Associates; 1998. p. 258.

51. Ganz PA, Coscarelli A, Fred C, Kahn B, Polinsky ML, Petersen L. Breast cancer survivors: psychosocial conmcerns and quality of life. Breast Cancer Res Treat. 1996;38:183-99.

52. Fobair P, Stewart SL, Chang S, D'Onofrio C, Banks P, Bloom JR. Body image and sexual problems in young women with breast cancer. Psychooncol. 2006;15:579-94.
53. Dany L, Apostolidis T, Cannone P, Suarez-Diaz E, Filipetto F. Body image and cancer: a psychosocial analysis. Psycho-Oncologie. 2009;3:101-17.

54. Harrington JM, Jones EG, Badger T. Body image perceptions in men with prostate cancer. Oncol Nurs Forum. 2009;36:167-72.

55. Reimers TS, Mortensen EL, Nysom K, Schmiegelow K. Healthrelated quality of life in long-term survivors of childhood brain tumors. Pediatric Blood Cancer. 2009;53:1086-91.

56. Denlinger CS, Barsevick AM. The challenges of colorectal cancer survivorship. J Natl Comprehensive Cancer Network. 2009; 8:883-93.

57. Jim HSL, Jacobsen. Posttraumatic stress and posttraumatic growth in cancer survivorship: a review. Cancer Journal. 2008;14:414-9. 